Rev. Elev. Méd. vét. Pays trop., 1975, 28 (3) : 419-425

\title{
Tourteau d'arachide détoxifié dans l'alimentation des volailles
}

\author{
par D. FRIOT $(*), H$. CALVET $\left({ }^{*}\right)$, S. DIALlO $(*)$ et M. WANE $\left({ }^{*}\right)$
}

\section{RESUME}

L'utilisation comparée dans l'alimentation de poulettes et de poussins de tourteau contenant de l'aflatoxine et de tourteau purifié par un procédé alcalin a montré que ce dernier donnait une production d'œufs plus importante, un taux d'éclosion meilleur, les poids et taux de consommation chez les adultes étant par ailleurs identiques pour les deux provendes.

\section{INTRODUCTION}

Le tourteau d'arachide, comme nombre d'autres produits, est susceptible d'être contaminé par une toxine, mise en évidence et appelée aflatoxine par les chercheurs anglais du Tropical Products Institue en 1960.

Elle est produite par une moisissure l'Aspergillus flavus qui se développe sur l'arachide avec une intensité plus ou moins grande suivant la pluviométrie, les conditions de culture et surtout de récolte.

Dès son isolement, cette toxine a suscité dans le monde entier une somme importante de travaux en raison des inconvénients que sa présénce dans l'alimentation peut provoquer chez de nombreuses espèces animales et également parce qu'on a pu la retrouver dans certaines productions zootechniques, le lait, où elle constitue alors un danger pour l'homme. L'aflatoxine en effet, chez certaines espèces, le rat en particulier, s'est comporté comme un facteur cancérigène.

Devant ces faits, les importateurs de tourteaux, préoccupés de la salubrité des produits,

(*) Laboratoire de Recherches Vétérina1res et Zootechniques, B.P. 2057 Dakar-Hann, République du Sénégal. ont fixé des normes plus ou moins sévères concernant les taux de contamination.

De leur côté, les pays producteurs d'arachide, par la mise au point de techniques culturales ou de méthodes de récolte adaptées, se sont également efforcés de diminuer la contamination de leurs produits.

Les industriels enfin ont recherché des procédés visant à assainir plus ou moins complètement les tourteaux. Le traitement à l'ammoniac entre autres s'est avéré efficace mais trop onéreux pour être vulgarisé. Des techniques utilisant les alcalins ont également été préconisées et c'est à un de ces procédés que s'est intéressée l'usine SODEC de Lyndiane au Sénégal. Il était alors indispensable de vérifier que du fait de ce traitement le tourteau ne subissait aucune altération capable d'en diminuer la valeur alimentaire, l'appétence ou, à l'extrême, de la rendre nuisible à la santé de l'animal.

Une expérimentation a été réalisée en 1974, dans ce sens. Elle utilise deux lots de poulettes pondeuses. Le choix de cette espèce se justifie par les raisons suivantes:

a) Possibilité d'utiliser dans la provende de fortes proportions de tourteau (18 à 20 p. 100 de protéines brutes suivant le stade de production); 
b) Sensibilité de cette espèce aux déséquilibres alimentaires ou aux intoxications quelle qu'en soit la nature;

c) Possibilité d'utiliser, pour tester la réaction, un nombre important de critères d'une grande sensibilité : poids, ponte (nombre et poids des œufs); taux d'éclosion des œufs mis en incubation; croissance des poussins.

d) Possibilité de mettre en expérience des lots homogènes comportant un nombre élevé d'individus, ce qui facilite l'interprétation statistique des résultats.

\section{CONDITIONS EXPERIMENTALES}

\section{Les animaux}

Les animaux d'expérience sont constitués de deux lots de 50 poulettes et 5 coquelets de race Leghorn, âgés de 7 à 8 mois et entrant juste en production, qui sont disposés dans des parquets de plein air, équipés de mangeoires, d'abreuvoirs, d'un abri nocturne et de pondoirs permettant une récolte rationnelle des œufs.

Composition des provendes (en pourcentage) tableau $\mathrm{n}^{0} \mathrm{I}$

Ces aliments ont fait l'objet de plusieurs séries d'analyses chimiques portant sur l'aliment complet, sur les tourteaux utilisés et sur leur teneur en aflatoxine.

\section{Analyse bromatologique des aliments complets}

Les résultats moyens de trois séries de dosages sont présentés dans le tableau $\mathrm{n}^{0} \mathrm{II}$;

Les valeurs théoriques de ces deux provendes sont par kg d'aliment : Energie métabolisable : provende pondeuses : 2635,7 ; provende pous-
TABLEAU $\mathrm{N}^{\circ} \mathrm{I}$

Composition des provendes (en p.100)

\begin{tabular}{|l|c|c|}
\hline \multirow{2}{*}{ Sorgho } & Poules & Poussins \\
\cline { 2 - 3 } Maïs & 25 & 25 \\
Rémoulage & 28 & 26 \\
Farine de poisson & 18 & 18 \\
Tourteau (A ou D) & 6 & 9 \\
Bicalcique & 15 & 20 \\
Carbonate de calcium & 1,2 & 1,2 \\
Sel & 6,1 & 0,55 \\
Vitamines + Oligo-êlëments & 0,45 & 0,25 \\
\hline
\end{tabular}

sins : 2896,6 . Matières protéiques digestibles : provende pondeuses: 163,8 ; provende poussins : 200,1; Rapport phosphocalcique : provende pondeuses : 0,34 ; provende poussins : 0,78 .

\section{Les tourteaux}

On constate que les différences concernant la composition des deux tourteaux portent

TABLEAU N$N^{\circ}$ III

Analyse bromatologique des deux tourteaux

\begin{tabular}{|l|r|r|}
\hline \multicolumn{1}{|c|}{ Eléments dosés } & Tourteaux D & Tourteaux N \\
\hline $\begin{array}{l}\text { Matières sèches g/kg } \\
\text { pouds brut } \\
\text { Matières minérales } \\
\text { g/kg poids sec } \\
\text { Matières grasses g/kg } \\
\text { poids sec }\end{array}$ & 857,3 & 885,4 \\
$\begin{array}{l}\text { Matières protéiques } \\
\text { g/kg poids sec }\end{array}$ & 703,5 & 64,8 \\
Cellulose Weende g/kg \\
paids sec \\
E N A g/kg poids sec \\
$\begin{array}{l}\text { Phosphore g/kg poids sec } \\
\text { Calcium g/kg poids sec }\end{array}$ & 532,3 & 59,7 \\
\hline
\end{tabular}

TABLEAU $N^{\circ}$ II

\begin{tabular}{|c|c|c|c|c|}
\hline \multirow[b]{2}{*}{ E 1 é me n t s d o s ês } & \multicolumn{2}{|c|}{$P \circ u 1$ e $s$} & \multicolumn{2}{|c|}{ P Oussins } \\
\hline & Aliment D & Aliment $\mathrm{N}$ & Aliment D & Aliment $\mathbb{N}$ \\
\hline Matières sèches en $\mathrm{g} / \mathrm{kg}$ poids brut & 896,4 & 900,0 & 885,0 & 900,1 \\
\hline Matıẽres minérales $\mathrm{g} / \mathrm{kg}$ poiơs $\mathrm{sec}$ & 116,2 & 113,5 & 66,6 & 62,5 \\
\hline Matières grasses $\mathrm{g} / \mathrm{kg}$ poids sec & 73.0 & 71,7 & 74,5 & 75,0 \\
\hline Matières protéiques $\mathrm{g} / \mathrm{kg}$ pords sec & 217,0 & 218,0 & 265,2 & 277,8 \\
\hline Matières cellulosiques (Weende) & 45,4 & 44,6 & 55,7 & 54,5 \\
\hline E N A g/ $/ \mathrm{kg}$ poids sec & 548,4 & 552,2 & 538 & 530,2 \\
\hline Phosphore & 11,1 & 10,0 & 10,8 & 10,1 \\
\hline Calcium & 33,0 & 36,0 & 11,2 & 11,8 \\
\hline
\end{tabular}

$D=$ détoxifıé ; $N=$ normal. 
essentiellement sur le taux de matières minérales sensiblement plus élevé dans le tourteau détoxifié $D$. Dans ce même tourteau, le taux de matières protéiques semble légèrement inférieur.

\section{Dosages de l'aflatoxine}

La méthode de dosage utilisée est la méthode désormais classique de l'AOAC adoptée par la CEE.

TABLEAU $\mathrm{N}^{\circ} \mathrm{IV}$

Résultats des 3 séries de dosage d'aflatoxine

\begin{tabular}{|c|c|c|c|c|}
\hline & $25 / 8 / 74$ & $22 / 10 / 74$ & $18 / 12 / 74$ & $\overline{\mathrm{x}}$ \\
\hline Tourteaux D & $160 \mathrm{ppb}$ & $100 \mathrm{ppb}$ & $110 \mathrm{ppb}$ & $\neq \neq 120 \mathrm{ppb}$ \\
\hline Tourteaux $\mathrm{N}$ & 1220 & $1250 \quad 11$ & $1070 "$ & 1180 \\
\hline
\end{tabular}

A l'imprécision de l'analyse près, on constate que les teneurs en aflatoxine du tourteau n'ont pas changé avec le temps et, d'autre part, que le procédé de détoxification est efficace, les teneurs en aflatoxine étant considérablement abaissées par le traitement.

\section{Déroulement de l'expérimentation et mesures effectuées}

L'expérimentation a été mise en place au début de juillet 1974 et s'est poursuivie jusqu'au 16 décembre de la même année.

Les volailles ont été pesées par lot chaque quinzaine; l'évaluation de la consommation avec établissement des refus deux fois par semaine est également hebdomadaire; les aufs sont récoltés chaque jour. Leur production est comptabilisée et pesée chaque semaine.

Quatre séries d'incubation ont été réalisées à partir du mois d'août comportant au total la mise en couveuse de 400 ceufs de $\mathrm{D}$ et 400 œufs de $\mathrm{N}$. Les poussins éclos ont été mis dans des éleveuses chauffées aux infrarouges et leur croissance surveillée par une pesée hebdomadaire. Des mesures de consommation ont été également effectuées chez ces jeunes.

\section{RESULTATS}

\section{Evolution du poids des adultes}

L'évolution du poids des adultes est représentée par le graphique A. Son examen montre que la courbe représentant l'évolution pondérale du lot $\mathrm{D}$ se situe pendant les $3 / 4$ de l'expérience au-dessus de celle du lot $\mathrm{N}$.

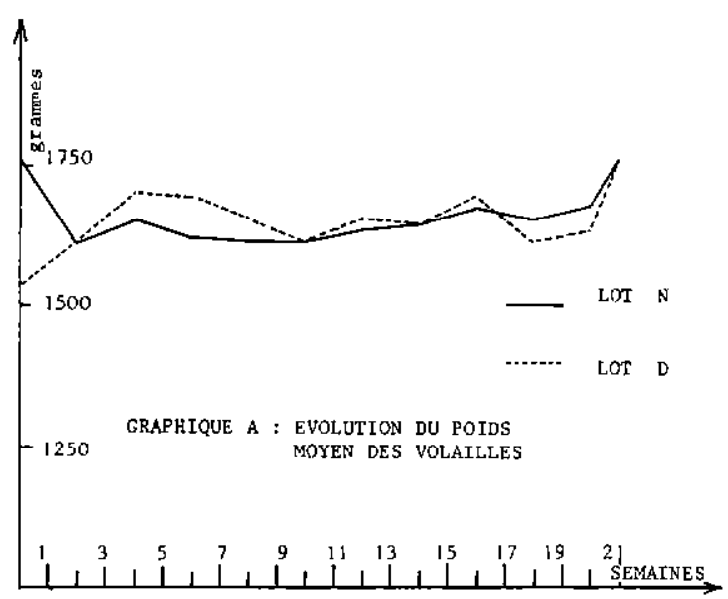

L'analyse statistique des résultats a montré que les poids moyens des volailles des lots $\mathrm{D}$ et $\mathrm{N}$ ne diffèrent pas. Les provendes $\mathrm{D}$ et $\mathrm{N}$ ont des actions identiques sur le poids des animaux.

\section{Consommation dans chaque lot}

Les chiffres concernant la consommation relevée dans chaque lot sont présentés par le graphique B qui montre l'évolution de cette consommation en $\mathrm{g}$ d'aliment par $\mathrm{kg}$ de poids vif de volaille.

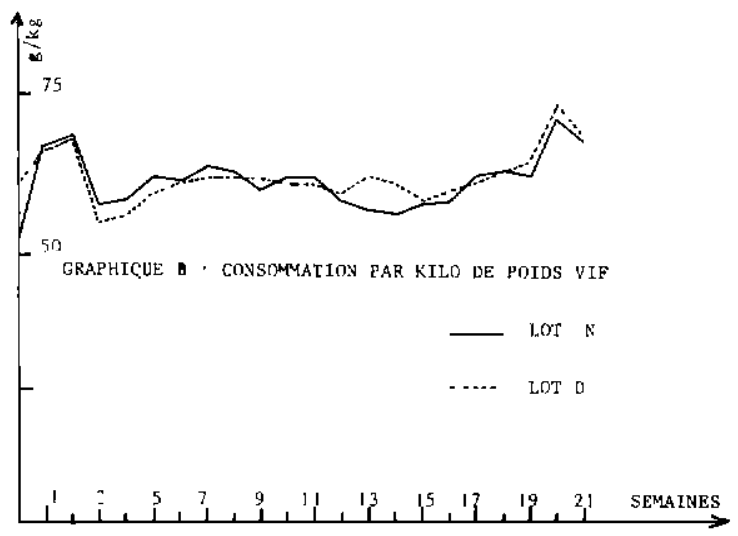

Il est important de savoir si la provende D, qui contient du tourteau détoxifié par traitement aux alcalins est mieux ou plus mal consommée que la provende contenant du tourteau d'arachide naturel. Des calculs ont été effectués d'une part sur la consommation par jour et par animal et d'autre part sur la consommation par $\mathrm{kg}$ de poids vif, par jour, pour éliminer l'influence du poids vif.

Des résultats obtenus, il ressort qu'il n'existe pas de différence significative entre les lots, 
quelle que soit la manière dont on exprime cette consommation.

En conséquence, le traitement chimique subi par le tourteau D n'entraîne aucun facteur d'inappétence par rapport à la provende contenant des tourteaux naturels.

\section{Ponte}

Durant la totalité de l'expérimentation, le nombre total des cufs récoltés a été :

- Lot D $=4992$ unités

- Lot $N=4690$ unités

Ce qui a conduit à une production en poids différent dans chaque lot:

- Lot $\mathrm{D}=276,68 \mathrm{~kg}$

- Lot $\mathrm{N}=267,00 \mathrm{~kg}$

\section{TAUX D'ECLOSION}

Les résultats ont été les suivants :

TABL. $\mathrm{N}^{\circ} \mathrm{V}$-Taux d'éclosion

\begin{tabular}{|c|c|c|c|c|}
\hline Lot & $\begin{array}{c}\text { Nombre } \\
\text { oeufs mis en } \\
\text { incubation }\end{array}$ & Clairs & $\begin{array}{r}\text { Nombre } \\
\text { d'oeufs } \\
\text { éclos }\end{array}$ & $\begin{array}{c}\text { Taux } \\
\text { d'êclosion }\end{array}$ \\
\hline & \multicolumn{4}{|c|}{ Eclosions d'Août } \\
\hline $\mathrm{D}$ & 200 & 6 & 157 & 78,5 \\
\hline $\mathrm{N}$ & 200 & 8 & 127 & 63,5 \\
\hline & \multicolumn{4}{|c|}{ Eclosions Novembre } \\
\hline $\mathrm{D}$ & 198 & 5 & 105 & 53 \\
\hline $\mathbf{N}$ & 200 & 10 & 78 & 39 \\
\hline
\end{tabular}

En définitive, au cours des différentes séries d'incubation, la proportion des poussins nés viables par rapport au nombre d'œufs mis en couveuse pour les différents lots s'établit comme suit :

Incubation d'août

- Lot $\mathrm{D}=78,5$ p. 100

- Lot $\mathrm{N}=63,5$ p. 100

Incubation de novembre

- Lot $\mathrm{D}=53$ p. 100

- Lot $\mathrm{N}=39$ p. 100

Par l'application du test de "l'écart réduit ", on constate que les taux d'éclosions du lot D sont nettement supérieurs à ceux du lot $\mathrm{N}$.

D'autres méthodes statistiques ont été utilisées pour comparer notamment la répartition des œufs clairs, morts en coquilles, morts après éclosion, éclos vivants dans les deux lots par des tests du $\chi^{2}$. Quelle que soit la manière de traiter le problème, la conclusion est toujours restée la même: taux d'éclosion de $D$ supérieur à $\mathrm{N}$.
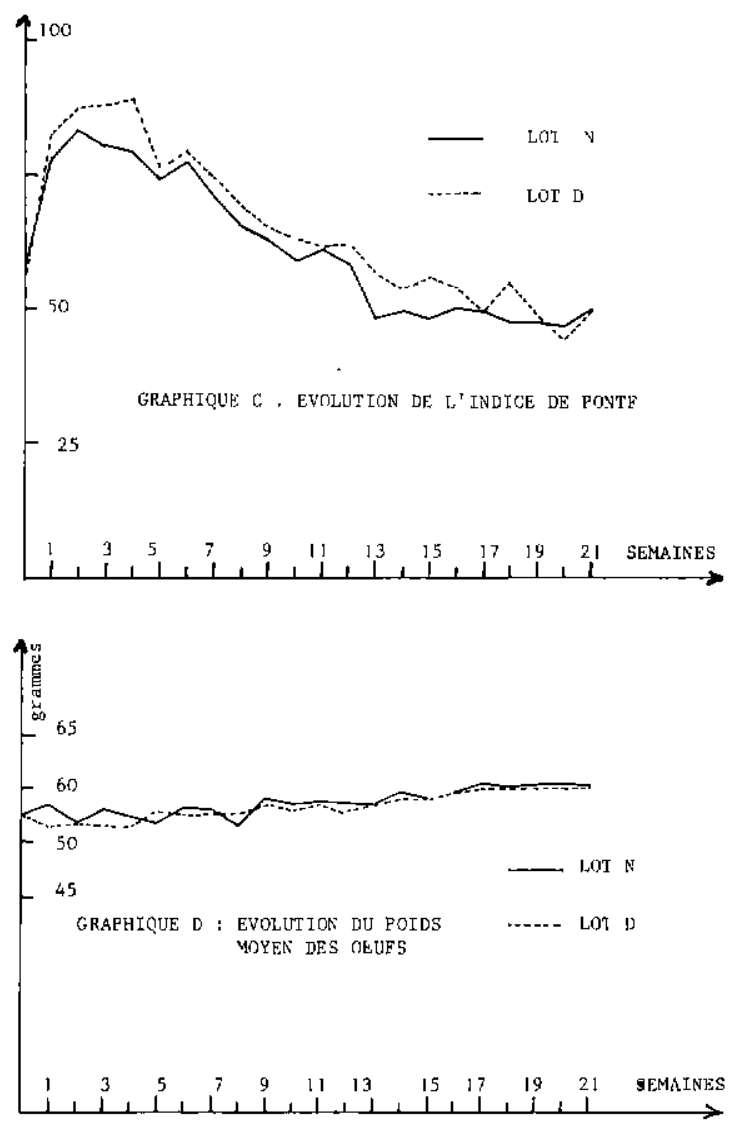

\section{CROISSANCE DES POUSSINS}

Les résultats observés sont donnés par les graphiques $\mathrm{E}$ et $\mathbf{F}$

\section{a) Eclosions d'août}

Pour cette période, la croissance hebdomadaire moyenne d'un poussin recevant du tourteau détoxifié est de $45,7 \mathrm{~g}$ alors que, pour une durée identique, cette croissance a été de $50,4 \mathrm{~g}$ pour le poussin recevant du tourteau non détoxifié.

\section{b) Eclosions de novembre}

Pour cette éclosion, les chiffres obtenus ont été respectivement de 38,0 et $39,9 \mathrm{~g}$.

L'analyse statistique par la méthode des blocs montre que, pour les deux périodes envi- 

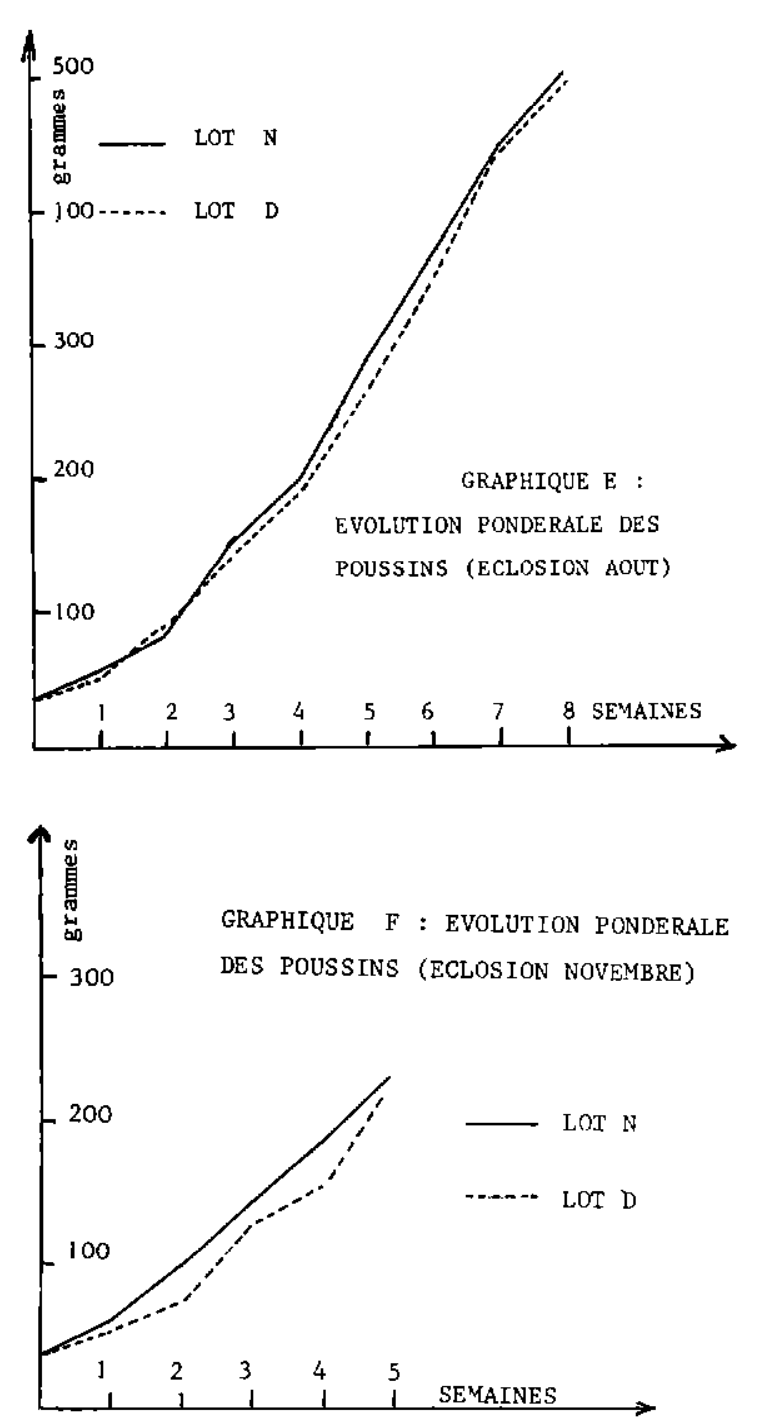

sagées, la croissance des poussins $\mathrm{N}$ est significativement supérieure à celle des poussins $\mathrm{D}$.

$\mathrm{Au}$ bout de huit semaines, le poids moyen du poulet $N$ est supérieur de 2 p. 100 à celui du poulet $\mathrm{D}$ pour l'éclosion d'août et de $4,4 \mathrm{p}$. 100 au bout de cinq semaines pour l'éclosion de novembre.

En définitive pour l'ensemble des éclosions, la croissance moyenne par semaine s'établit ainsi pour chaque lot:

- Lot D : 41,8 g

- Lot $\mathrm{N}: 45,1 \mathrm{~g}$

A ce niveau, ce sont donc les poussins recevant du tourteau normal qui semblent reprendre l'avantage sur ceux du lot recevant du tourteau détoxifié.

\section{Consommation des poussins}

Les résultats des calculs sur la consommation des poussins par $\mathrm{kg}$ de poids vif et par jour montrent qu'elle est identique dans les deux lots. Les provendes $\mathrm{D}$ et $\mathrm{N}$ sont appétées de façon égale par les poussins.

Résultats synthétiques des comparaisons statistiques.

a) L'évolution des poids dans les deux lots de volailles adultes est comparable.

b) Le lot $\mathrm{D}$ recevant le tourteau détoxifié a eu un taux de ponte supérieur à celui du lot $\mathrm{N}$.

c) Le poids total des cufs pondus par le lot $\mathrm{D}$ est plus élevé que celui des œufs récoltés dans le lot $\mathrm{N}$.

d) L'œuf du lot $\mathrm{N}$ est plus lourd que l'œuf du lot D.

e) Le taux d'éclosion du lot $\mathrm{D}$ est nettement supérieur à celui du lot $\mathrm{N}$.

f) La croissance des poussins dans le lot $\mathrm{N}$ est supérieure à celle observée dans le lot $\mathrm{D}$.

g) La consommation tant chez les adultes que chez les jeunes a été comparable dans les deux lots.

\section{DISCUSSIONS ET CONCLUSIONS}

Le procédé de détoxification des tourteaux utilisé par la SODEC a démontré son efficacité, comme en témoignent les séries de dosage d'aflatoxine effectuées :

Taux de contamination moyen du tourteau traité : $120 \mathrm{ppb}$

Taux de contamination moyen du tourteau non traité : $1200 \mathrm{ppb}$.

Le taux de contamination tolérable dans les aliments destinés au bétail tel qu'il est défini par les normes de la C.E.E. est de $50 \mathrm{ppb}$ dans les aliments composés. Le tourteau normal incorporé par exemple dans une provende contenant 20 p. 100 de tourteau aurait entraîné un taux de contamination du mélange de $240 \mathrm{ppb}$ alors que l'incorportation du tourteau traité réduit le taux de contamination du mélange à $24 \mathrm{ppb}$, ce qui est admissible pour les normes provisoires actuelles. 
En faveur du tourteau détoxifié, sont apparus deux facteurs favorables importants: le taux de ponte et celui d'éclosion. On peut donc conclure à une plus grande efficacité dans la provende A dans le domaine de la production. II semble bien que ces deux faits expérimentaux importants soient en relation avec la diminution du taux d'aflatoxine dans les tourteaux traités.

L'analyse statistique a montré qu'individuellement les œufs provenant du lot A étaient d'un poids légèrement inférieur à ceux du lot $\mathrm{N}$. Il ne semble pas que cette légère différence puisse constituer un résultat expérimental. En effet, il est tout à fait dans l'ordre des choses qu'au sein d'une même race une plus grande fréquence de la ponte entraîne une diminution du poids des cufs.

\section{Critères défavorables}

La croissance des poussins du lot $\mathrm{D}$ a été inférieure à celle des poussins du lot $\mathrm{N}$.

Il ne semble pas que la légère différence observée dans l'analyse bromatologique des deux provendes en ce qui concerne en particulier les taux de matières minérales et de matières protéiques puisse avoir une responsabilité quelconque de ce fait.

Par contre une explication plus logique réside dans la différence du poids des ceufs notée précédemment. On sait en effet que dans la plupart des espèces, le poids de la naissance est un facteur important de la croissance du jeune tout au moins dans son début.

Les poules du lot $\mathbf{D}$ ont en définitive pondu davantage. Le poids des œufs s'en est trouvé réduit de même que celui des poussins à l'éclosion (différence de poids de 1,5 p. 100).

Il en est résulté une croissance moins bonne durant le premier mois d'élevage, ce qui se serait très probablement atténué par la suite.

En conclusion, on peut dire que le procédé de détoxification utilisé est efficace et que la détoxification industrielle des tourteaux paraît donc constituer une solution immédiate d'un des importants problèmes posés par la présence de l'aflatoxine dans les sous-produits de l'arachide.

\section{RESUME}

Une provende pour volailles contenant du tourteau d'arachide commun est comparé à une provende identique mais contenant du tourteau d'arachide détoxifié par un procédé alcalin abaissant le taux d'aflatoxine. L'expérimentation menée sur des poulettes et leurs poussins donne les résultats suivants pour le lot détoxifié : production d'oufs plus importante; taux d'éclosion meilleur; croissance des poussins plus faible; aucune différence de poids entre lots chez les adultes; aucune différence de consommation entre lots chez les adultes et chez les poussins.

Le procédé de détoxification est efficace et les effets sur les volailles du tourteau obtenu semblent bénéfiques.

\section{SUMMARY}

\section{Detoxificated groundnut oil cake in poultry feeding}

\footnotetext{
A poultry-food consisting of 15 to 20 percent of common groundnut oil-cake was compared to a poultry food alike but containing a groundnut oil-cake detoxificated through an alkaline process aiming to decrease the aflatoxin amount. The experiment was carried out on hens and their chicks. The following results with the detoxificated oil-cake were obtained :

- increased egg-production;

- improved hatching;

- delayed growth of chicks;

- no significative weight difference between the two batches of hens;

- no consumption difference between batches among adults and chicks.

The oil-cake detoxification process seems efficient and the effects on poultry production favourable.
} 


\title{
RESUMEN
}

\section{Torta de cacahuete detoxificado para la alímentación}

\author{
de las aves de corral
}

\begin{abstract}
Se compara un pienso para aves de corral conteniendo torta de cacahuete común con un pienso idéntico pero conteniendo torta de cacahuete detoxificado por un procedimiento alcalino bajando la tasa de aflatoxina. La experimentación hecha con lluecas y sus pollitos da los resultados siguientes en lo concerniendo al lote detoxificado: producción más importante de huevos; mejor tasa de rompimiento; crecimiento más leve de los pollitos; ninguna diferencia de peso entre los lotes en los adultos; ninguna diferencia de consumo entre lotes en los adultos y en los pollitos. El procedimiento de detoxificación es eficaz y los efectos sobre las aves de corral de la torta obtenjda parecen benéficos.
\end{abstract}

\section{BIBLIOGRAPHIE SUCCINCTE}

1. ALLCROFT (R.), LEWIS (G.). Groundnut toxicity in cattle; experimental poisoning of calves and a report on clinical effects in older cattle. Vet. Rec., 1963,75 (19) : 487-493.

2. ASPLIN (F. D.), CARNAGHAN (R. B. A.). The toxicity of certain groundnut meals for poultry with special reference to their effects on ducklings and chickens. Vet. Rec., 1961, 73 (46) : 1215-19.

3. AYCARDY (J.), CALET (C.). Manifestation de la toxicité d'échantillons contaminés par Aspergillus flavus chez les oiseaux de basse-cour. Industr. Alim. anim. Fr., 1966, 35 (177): 37.45.

4. BOUDERGUES (R.), CALVET (H.), DISCACCIATI (E.), CLICHE (M.). Note sur la présence d'aflatoxine dans les fanes d'arachide. Rev. Elev. Méd. vét. Pays trop., 1966, 19 (4) : 567-571.

5. BOUDERGUES (R.). Effets expérimentaux de l'aflatoxine sur des femelles d'espèce bovine et sur leurs veaux. Thèse doct. Univ. Toulouse. Faculté mixte de Médecine et de Pharmacie, 1968.

6. CALVET (H.), BOUDERGUES (R.), DISCAC. CIATI (E.), CLICHE (M.). Note préliminaire sur les effets expérimentaux d'aflatoxine chez les bovins tropicaux. Rev. Elev. Méd. vét. Pays trop., 1966, 19 (4) : 545-565.

7. COOMES (T. J.), CROWSTHER (P. C.), FEUELL (A. J.), FRANCIS (B. J.). Experimental detoxification of groundnut meals containing aflatoxin. Nature, London, 1966, 209 : 406-407.

8. PLATT (B.S.), STEWART (R. J.C.), GUPTA (R.). Toxicity of aflatoxin for chick embryos in fertilized eggs. Proc. Natr. Soc., 1962, 30 : 21.

9. VORSTER (L. J.). Etude sur la détoxification des arachides contaminées par l'aflatoxine et destinées à l'huilerie. Rev. Fr. Corps gras, 1966, 13 (1) : 7-12. 